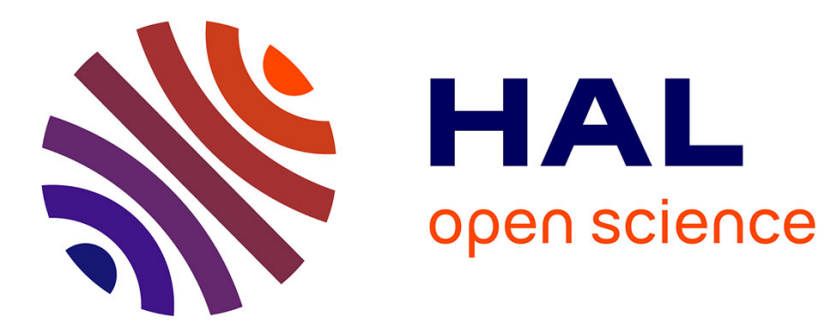

\title{
Motion-based prediction of hands and feet contact efforts during asymmetric handling tasks
}

\author{
Antoine Muller, Charles Pontonnier, Georges Dumont
}

\section{To cite this version:}

Antoine Muller, Charles Pontonnier, Georges Dumont. Motion-based prediction of hands and feet contact efforts during asymmetric handling tasks. IEEE Transactions on Biomedical Engineering, 2020, 67 (2), pp.344-352. 10.1109/TBME.2019.2913308 . hal-02109407

\section{HAL Id: hal-02109407 \\ https://hal.inria.fr/hal-02109407}

Submitted on 24 Apr 2019

HAL is a multi-disciplinary open access archive for the deposit and dissemination of scientific research documents, whether they are published or not. The documents may come from teaching and research institutions in France or abroad, or from public or private research centers.
L'archive ouverte pluridisciplinaire $\mathbf{H A L}$, est destinée au dépôt et à la diffusion de documents scientifiques de niveau recherche, publiés ou non, émanant des établissements d'enseignement et de recherche français ou étrangers, des laboratoires publics ou privés. 


\title{
Motion-based prediction of hands and feet contact efforts during asymmetric handling tasks
}

\author{
Antoine Muller, Charles Pontonnier, and Georges Dumont
}

\begin{abstract}
This paper proposes a method to predict the external efforts exerted on a subject during handling tasks, only with a measure of his motion. These efforts are the contacts forces and moments on the ground and on the load carried by the subject. The method is based on a contact model initially developed to predict the ground reaction forces and moments. Discrete contact points are defined on the biomechanical model at the feet and the hands. An optimization technique computes the minimal forces at each of these points satisfying the dynamic equations of the biomechanical model and the load. The method was tested on a set of asymmetric handling tasks performed by 13 subjects and validated using force platforms and an instrumented load. For each task, predictions of the vertical forces obtained a RMSE of about $0.25 \mathrm{~N} / \mathrm{kg}$ for the feet contacts and below $1 \mathrm{~N} / \mathrm{kg}$ for the hands contacts. L5/S1 joint moments were then computed using the predicted and the measured data. RMSE of $18 \mathrm{Nm}$ and rRMSE below $10 \%$ were obtained for the flexion/extension component. In conclusion, this method enables to quantitatively assess asymmetric handling tasks on the basis of kinetics variables without additional instrumentation such as force sensors and thus improve the ecological aspect of the studied tasks. This method has a great potential to be applied in work tasks analyses in ergonomics studies or sports gestures analyses involving hand contacts in exercise science.
\end{abstract}

Index Terms-Ergonomics, optimization, dynamics, ground reaction forces, neural network.

\section{INTRODUCTION}

$\mathbf{I}^{\mathrm{s}}$ NVERSE dynamics is a classical analysis tool used to study work tasks at a biomechanical level. In particular handling tasks are one of the core tasks to study in workplace ergonomics since this is one of the most constraining motion to realize in a work situation [1]. Indeed, these tasks are particularly prone to musculoskeletal disorders. In such tasks, the studied

A. Muller is with the Institut de recherche Robert-Sauvé en santé et en sécurité du travail (IRSST), Montréal, QC, Canada

C. Pontonnier and G. Dumont are with the Univ Rennes, CNRS Inria, IRISA - UMR 6074, M2S, 35042 Rennes, France biomechanical variables are based on the kinematics (for example the postural asymmetries or the lumbar spine flexion) and based on the kinetics (for example forces and moments in the lumbar spine [2]-[4]).

In addition to motion capture data used to compute the kinematics variables, estimation of joint moments and muscle forces requires the knowledge of the contact forces. Most of the studies concerning handling tasks are based on a bottom-up approach using force platforms to measure the ground reaction forces and moments (GRF\&M). The use of such devices affects the ecological aspect of the tasks by constraining the field of acquisition to the force platforms. Similarly, the instrumentation of the loads with force sensors limits the tasks to be studied since the subject must only use instrumented handles.

Numerous studies predicted the external efforts using only motion data. Generally, the external efforts were limited to the GRF\&M. The use of dynamics equations with the application of a top-down approach is limited by the fact that the inverse dynamics problem is undetermined during the double support phase. Three approaches were proposed in the literature to solve this issue: empirical functions [5], [6], machine learning [7], [8] and contact models.

First, empirical functions were used to distribute the weight under the feet [5]. A method based on the zero moment point was proposed to predict the point of application acting of each foot [6]. This approach is very simple to apply but is limited to the GRF\&M prediction on gait motion.

Second, the use of machine learning methods was proposed on static posture, gait and asymmetric movements [7], [8]. The estimation of GRF\&M for one side was carried out with a neural network and by using the dynamics equations on the other side. On the same basis, a set of sidestep motions was used to establish a link 
between the motion and the GRF\&M [9]. Then, the use of a regression method allowed the GRF\&M to be predicted using only the motion. These methods, based on a learning phase, have the disadvantage to require a considerable set of motions representative to the studied one and thus are only applicable to standardized tasks.

Third, a contact model with optimization techniques was used to solve the indeterminacy [10]-[12]. The method added a set of artificial muscle-like actuators under each foot of a musculoskeletal model. These additional actuators were considered in a similar way as the muscles of the model in the muscle recruitment algorithm. The optimization problem found muscle forces which minimize a cost function by satisfying the dynamics equations. The GRF\&M corresponded to the forces contained in the artificial muscle-like actuators. A validation on activities of daily living and on sports-related movements was done [10]-[12]. However, this approach was not validated on handling tasks and was limited to the estimation of GRF\&M.

Prediction of external efforts with other contacts than feet on the ground was applied to sit-to-stand tasks [13], [14]. The contacts were feet, hands and buttocks. On a planar study, each of the 3 contact points was associated to a force and a moment. Based on the same idea as defined before, an optimization problem computed the external efforts by minimizing a cost function and by satisfying the dynamics equations. However, this work was limited to a 2D study. Handling tasks composed of a large part of asymmetric movements should be analyzed with a 3D study. Elsewhere, load contact forces and moments (LCF\&M) were predicted based on the motion during symmetrical lifting tasks [15]. However, GRF\&M were measured through force platforms in this study.

Thus, the aim of the current paper is to develop a prediction method of the GRF\&M and the LCF based only on the subject motion during asymmetric handling tasks. This method estimates kinetics variables such as back loading during handling tasks based only on subject motion, improving significantly the ecological aspect of the experiments. The method proposes an estimation of the GRF\&M and LCF based on optoelectronical motion capture data. Prior to this estimation, a step of identification of grip and deposit events and a step of load motion reconstruction are applied. The validation consists in, first, comparing the predicted GRF\&M and the LCF to the measured ones and second, comparing predicted back loading (evaluated as the L5/S1 joint moments) to those computed from measured data. The results are detailed and discussed at the end of the paper.

\section{MATERIALS AND METHODS}

\section{A. Experimental procedures}

13 male participants (age: $27 \pm 7$ years old, height: $177 \pm 4 \mathrm{~cm}$, mass: $73 \pm 15 \mathrm{~kg}$ ) participated in this study. All subjects signed an informed consent form before participation and an anonymization protocol was followed for data safeguarding. This study has been approved by a National Ethics Committee (Comité de Protection des Personnes, 2018-A00484-51). The task required to carry a load from one area to another. A set of three elementary movements was consider as a cycle. The first one was from area 1 to area 2, the second one from area 2 to area 3 and the third one from area 3 to area 1 (Figure 1). The subject was asked to carry the load with the handles at a self-selected speed. The subject was also free to lift and move his feet as long as each foot stayed in contact with the associated platform (further details about the force platforms in the next section). Between every elementary movement, the subject went back to the initial position.

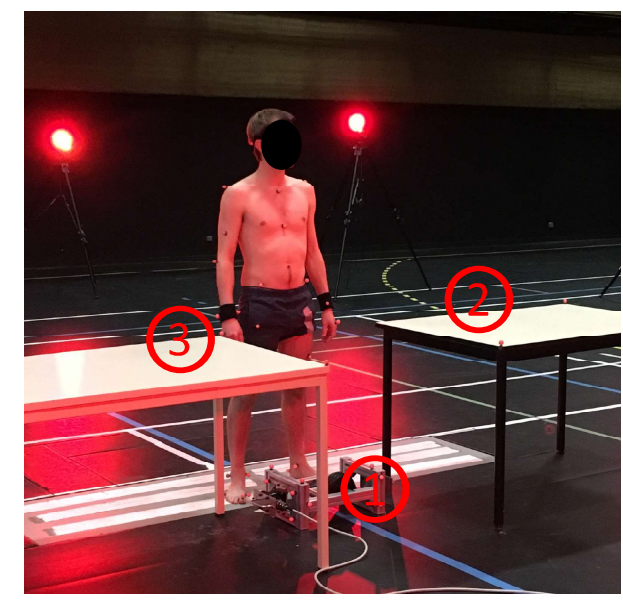

Figure 1: Experimental protocol used for the asymmetric handling tasks.

In the experiments, the load to carry was a custom instrumented load $(42 \mathrm{~cm} \times 27 \mathrm{~cm} \times 18 \mathrm{~cm})$ of $6.9 \mathrm{~kg}$ equipped with two handles (Figure 2). A 6-axis force sensor allowed to measure the efforts at the contact of one of the hands. Additional loads could be added to vary the inertial parameters of the load (mass, position of the center of mass and inertia) resulting in different experimental conditions. 


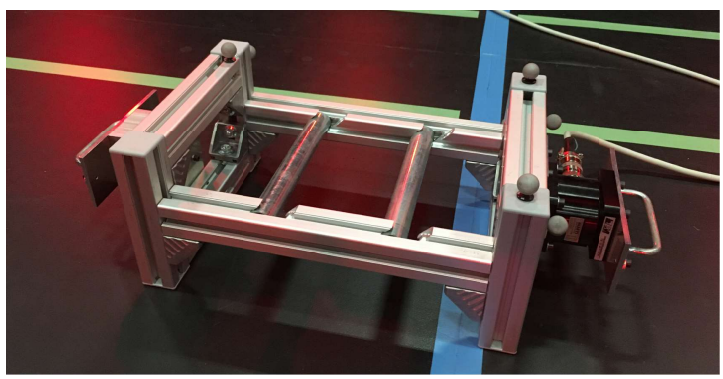

Figure 2: Custom instrumented load used in the experiments. A 6-axis force sensor measured the efforts applied by one of the hands.

Each subject completed two cycles with the standard load and two cycles with an additional load of $3 \mathrm{~kg}$.

The experimental setup was composed of 47 motion capture markers (Figure 1) placed on standardized anatomical landmarks as recommended by the International Society of Biomechanics [16], [17]. Two markers were placed on each hand (third and fifth metacarpal) to improve their kinematics reconstruction. The location of these markers were chosen in order to minimize the discomfort of the subjects. Motion capture markers were recorded with a 16 cameras Vicon $^{\circledR}$ motion capture system at $200 \mathrm{~Hz}$. Subjects stood over two AMTI ${ }^{\circledR} 120 \times 60 \mathrm{~cm}$ force platforms $(1000 \mathrm{~Hz})$, each supporting one foot. Nine additional markers were placed on the instrumented load to reconstruct its motion for the validation part (Figure 2). All analog data acquisition was synchronized with the motion capture data using a single Vicon MX giganet box to collect the data and Nexus 2.6.1. to configure the synchronous channels.

The external efforts prediction method proposed in this study used only the position of the subject markers as experimental data. It also required additional parameters as the size and the mass of the subject and the inertial parameters of the load. The validation of the method used the position of the markers on the load, the GRF\&M measured by the two force platforms, the LCF\&M measured by the 6 -axis force sensor and the video of the experiments.

\section{B. External efforts prediction}

The external efforts acting on the subject during the tasks were the GRF\&M and, when a load was carried, the LCF\&M. The proposed method was composed of three successive steps:
1) detection of grip and deposit events to identify if the load was carried by the subject or not;

2) load motion reconstruction between a grip and a deposit event;

3) external efforts prediction based on the equations of motion applied to the subject or simultaneously to the subject and the load.

The first two steps used only the positions of one marker on each hand. The third step used all the markers located on the subject.

1) Detection of grip and deposit events: The knowledge of the grip and deposit events was crucial since it allowed to distinguish phases where external efforts were applied on the hands or not. A statistical method based on a neural network has been used to evaluate, at any time, the probability to have a grip or a deposit event.

The neural network was only based on the position over time of one marker located on top of the right hand and one marker located on the top of the left hand (both on the third metacarpal). Five descriptors were used: the distance between the two markers, the velocity and the acceleration of the first marker and the velocity and the acceleration of the second one. Considering the instant $t$ (allowing to evaluate the probability that this instant was an instant of grip or deposit), we used the input variables of a window of $N$ instants centered on the instant $t(N=$ 100 in this study). The velocities and the accelerations were computed with a 2 -order finite-difference method. To improve this computation, the marker positions were filtered with a 4-th order Butterworth low pass filter with a cut-off frequency of $5 \mathrm{~Hz}$ and no phase shift [18].

In the learning phase, the reference grip and deposit events $\left(G E_{r e f}\right.$ and $\left.D E_{\text {ref }}\right)$ were identified from a video record of the experiments. For each instant, the probability to have a grip or deposit event was defined by using a normal distribution centered on the reference events with a standard deviation $\sigma$ (Figure 3). After different tests, the value of $5 \sigma$ was selected considering that an instant at a distance of more than 1 second from a reference instant had no chance to be a grip or deposit event ( $5 \sigma$ corresponds to 1 second).

In the application phase, for a cycle containing one grip and one deposit event, the probability of a grip or deposit event at each instant was evaluated from the positions of markers on each hand with the neural network. The resulting curve was then filtered with a moving average of 100 frames (corresponding to 0.5 
Probability of a grip

or deposit moment

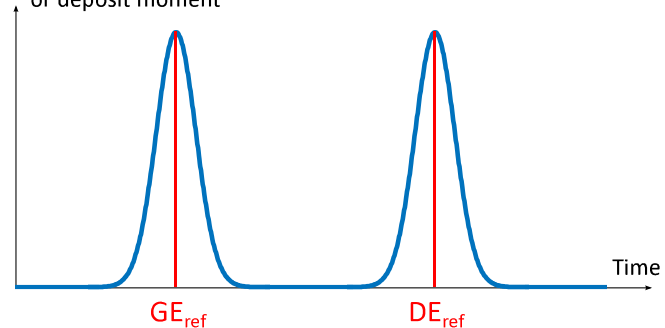

Figure 3: Probability distribution of a grip or deposit for each instant from the reference grip event $\left(G E_{r e f}\right)$ and from the reference deposit event $\left(D E_{r e f}\right)$.

second). The grip and deposit events were thus identified as the frames corresponding to the 2 peaks with the highest prominence.

2) Load motion reconstruction: The use of the equations of motion applied to the load required the knowledge of its motion. With a prospective view to assess handling tasks as close as possible that it is done in workplace, this measurement with markers would be very difficult. Indeed, handlers carry out several stacked loads which prevent the use of markers. In this view, the method was based only on the subject motion. The load markers were used for the validation. The load motion was reconstructed from the positions of one marker on the right hand and one marker on the left hand (the same as those used for the identification of grip and deposit moments). The load motion reconstruction consisted in defining a coordinate system associated to the load $\left(O_{l}, \boldsymbol{x}_{\boldsymbol{l}}, \boldsymbol{y}_{\boldsymbol{l}}, \boldsymbol{z}_{\boldsymbol{l}}\right)$. The geometrical center of the load $O_{l}$ was defined as the center of the two markers. The $z_{l}$ direction was associated to the direction between the two markers. Moreover, we assumed that, during the handling task, there was no rotation of the load around $z_{l}$. Thus, we defined $x_{l}$ as the projection of the vertical axis on a plane orthogonal to $z_{l}$. The $y_{l}$ definition allowed to define a direct orthonormal coordinate system.

3) External forces prediction: For this step, we used a biomechanical model composed of 18 rigid segments (pelvis, lower trunk, upper trunk, head, clavicles, upper arms, lower arms, hands, upper legs, lower legs and feet) linked by 17 joints corresponding to 41 degrees of freedom (3 for the pelvis/lower trunk joint, 3 for the lower trunk/upper trunk joint, 3 for the neck, 3 for each the upper trunk/clavicles joint, 3 for each shoulder, 2 for each elbow, 2 for each wrist, 3 for each hip, 1 for each knee and 2 for each ankle). The geometrical parameters were subject-specific calibrated [19]. Body segment inertial parameters (BSIP) were computed from [20]. The joint coordinates $\boldsymbol{q}$ were computed in an inverse kinematics step [21] and then filtered with a 4-th order Butterworth low pass filter with a cut-off frequency of $5 \mathrm{~Hz}$ and no phase shift [18]. The prediction method considered the external efforts applied on the feet (GRF\&M) and external efforts applied on the hands (LCF\&M). The equations of motion used in the prediction were those applied to the subject and those applied to the load.

We defined a set of discrete contact points on the biomechanical model: $N_{f}$ points under each foot and $N_{h}$ points on each hand. For this study, $N_{f}=14$ and $N_{h}=11$ were selected (Figure 4). These points were defined to map the contact area [10], [12]. Two phases were then distinguished: the subject carried the load or not. The detection of grip and deposit events allowed to distinguish them.

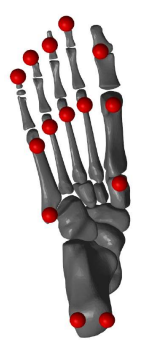

(a)

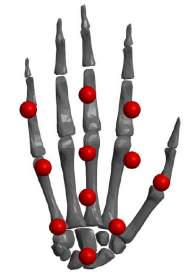

(b)
Figure 4: Discrete contact points defined on the biomechanical model. (a): 14 contact points under each foot; (b): 11 contact points on each hand.

When the subject did not carry the load, the prediction method was a classical GRF\&M prediction method from the equations of motion applied to the subject. Thus, at each instant, the external efforts were the solution of the optimization problem (1), solved with a Sequential Quadratic Programming (SQP) method.

$$
\begin{array}{ll}
\min _{\boldsymbol{F}} & \sum_{i=1}^{2 N_{f}}\left\|\boldsymbol{F}_{\boldsymbol{i}}\right\|^{2} \\
\text { s.t. } & \boldsymbol{M}_{\boldsymbol{s}}(\boldsymbol{q}) \ddot{\boldsymbol{q}}+\boldsymbol{C}_{\boldsymbol{s}}(\boldsymbol{q}, \dot{\boldsymbol{q}})+\boldsymbol{G}_{\boldsymbol{s}}(\boldsymbol{q})+\boldsymbol{\lambda}_{\boldsymbol{s}}+\boldsymbol{E}_{\boldsymbol{s}}=\mathbf{0} \\
& \forall i \in \llbracket 1,2 N_{f} \rrbracket, \boldsymbol{F}_{\boldsymbol{i}} \leq \boldsymbol{F}_{\boldsymbol{i}}^{\boldsymbol{m a x}}
\end{array}
$$

Where, by isolating the biomechanical model, $\boldsymbol{M}_{\boldsymbol{s}}(\boldsymbol{q})$ is the inertia matrix, $\boldsymbol{C}_{\boldsymbol{s}}(\boldsymbol{q}, \dot{\boldsymbol{q}})$ is the centrifugal and 
Coriolis force vector, $\boldsymbol{G}_{\boldsymbol{s}}(\boldsymbol{q})$ is the gravity force vector, $\lambda_{s}$ is the generalized internal force vector, $\boldsymbol{E}_{\boldsymbol{s}}$ is the generalized external force vector and $\boldsymbol{F}_{i}^{\max }$ is the vector containing the maximal forces available for the contact point $i$. At each instant, we ensured that each actuator was sufficiently close to the floor and almost without motion [10], [12]. The distance and velocity thresholds were respectively $0.02 \mathrm{~m}$ and $0.8 \mathrm{~m} / \mathrm{s}$. When a contact point was respecting the thresholds, the associated force was limited to $0.4 \mathrm{BW}$ (Body Weight) and had to respect the Coulomb's law of friction. A friction coefficient of 0.5 was used here [12], [14]. The external force vector $\boldsymbol{E}_{\boldsymbol{s}}$ contained the external efforts $\boldsymbol{F}_{\boldsymbol{i}}$ applied on the feet.

When the subject carried the load, the prediction method took into account simultaneously the equations of motion applied to the subject and the equations of motion applied to the load. Thus, at each instant, the external efforts were the solution of the optimization problem (2), solved with a SQP method.

$$
\begin{array}{ll}
\min _{\boldsymbol{F}} & \sum_{i=1}^{2\left(N_{f}+N_{h}\right)}\left\|\boldsymbol{F}_{\boldsymbol{i}}\right\|^{2} \\
\text { s.t. } & \boldsymbol{M}_{\boldsymbol{s}}(\boldsymbol{q}) \ddot{\boldsymbol{q}}+\boldsymbol{C}_{\boldsymbol{s}}(\boldsymbol{q}, \dot{\boldsymbol{q}})+\boldsymbol{G}_{\boldsymbol{s}}(\boldsymbol{q})+\boldsymbol{\lambda}_{\boldsymbol{s}}+\boldsymbol{E}_{\boldsymbol{s}}=\mathbf{0} \\
& \boldsymbol{M}_{\boldsymbol{l}}(\boldsymbol{q}) \ddot{\boldsymbol{q}}+\boldsymbol{C}_{\boldsymbol{l}}(\boldsymbol{q}, \dot{\boldsymbol{q}})+\boldsymbol{G}_{\boldsymbol{l}}(\boldsymbol{q})+\boldsymbol{E}_{\boldsymbol{l}}=\mathbf{0} \\
& \forall i \in \llbracket 1,2\left(N_{f}+N_{h}\right) \rrbracket, \boldsymbol{F}_{\boldsymbol{i}} \leq \boldsymbol{F}_{\boldsymbol{i}}^{\max }
\end{array}
$$

Where, by isolating the load, $\boldsymbol{M}_{\boldsymbol{l}}(\boldsymbol{q})$ is the inertia matrix, $C_{l}(\boldsymbol{q}, \dot{\boldsymbol{q}})$ is the centrifugal and Coriolis force vector, $\boldsymbol{G}_{\boldsymbol{l}}(\boldsymbol{q})$ is the gravity force vector and $\boldsymbol{E}_{\boldsymbol{l}}$ is the generalized external force vector. The maximal forces for contact points on the feet were the same as defined above. The external efforts vector $\boldsymbol{E}_{\boldsymbol{s}}$ contained the external forces applied on the feet and the external efforts vector $\boldsymbol{E}_{\boldsymbol{l}}$ contained the external forces applied on the hands. The predicted and the measured forces were filtered with a 4-th order Butterworth low pass filter with a cut-off frequency of $5 \mathrm{~Hz}$ and no phase shift.

The motion of the load used in the equations of motions described in equation (2) is the one obtained at the previous step. Inertial parameters of the load were estimated from its digital mock-up on which were applied the corresponding materials for both configurations (with and without the additional load).

\section{L5/S1 joint moments estimation}

L5/S1 joint moments were estimated by using the predicted and the measured data with a recursive NewtonEuler algorithm [22] and a bottom-up approach. As measured efforts were available only for one of the hands, the L5/S1 moments were computed by using the GRF\&M.

\section{Data analysis}

Motion decomposition: To facilitate the results analysis, each movement was separated into elementary handling motions, which were divided in three phases: the subject was not in contact with the load, the subject carried the load and the subject was in contact with the load but without completely carrying it. As the method is less efficient in this latter phase due to the lack of information about contacts at the hands, it is defined as the uncertainty phase thereafter. The different periods were identified with a video analysis of the tasks by finding the instants of the first and the last contact between the subject and the load and the instants of the first and the last contact between the load and the ground. The reference grip and deposit events (used in the learning phase of the neural network) were defined as the middle of the associated uncertainty phase.

Evaluation of the grip and deposit events detection: The identification of grip and deposit events contained a learning and an application phase. The method was validated with a leave-one-out cross validation. For each validation, all movements of one subject were excluded from the learning phase. It enabled to test if the method could be applied on a new subject (no participation to a previous study).

Evaluation of the load motion reconstruction: During phases where the subject carried the load, its motion reconstruction was compared to the motion of the markers placed on the load. The root-mean-square error (RMSE) of the distance and the orientation between the reconstructed and the markers-based coordinate systems was computed.

Evaluation of the external efforts prediction: Predicted GRF\&M were compared to those measured by the force platforms. The predicted LCF on the right hand were compared to the experimental data given by the 6axis force sensor. For each elementary movements the RMSE and the relative RMSE (rRMSE) (as defined 
by [23]) were computed to compare the experimental and the predicted data. To improve the comparison to results reported in the literature, RMSE of forces (and moments) are presented in $\mathrm{N}$ (and $\mathrm{Nm}$ ) and in $\mathrm{N} / \mathrm{kg}$ (and $\mathrm{Nm} / \mathrm{kg}$ ). Even if external efforts were predicted throughout the motion, the uncertainty phases were not taken into account in this analysis, as discussed in the last part of this paper.

Evaluation of the L5/S1 joint moments estimation: L5/S1 joint moments computed with the predicted and with the measured data were compared. RMSE and rRMSE [23] along each axis are presented.

All the data was processed with CusToM (Customizable Toolbox for Musculoskeletal simulation) in Matlab ${ }^{\circledR}$ [24], in which was implemented the GRF\&M prediction method.

\section{RESULTS AND DISCUSSION}

\section{A. Detection of grip and deposit events}

The Figure 5 shows a representative example of an identification of grip and deposit events for one elementary movement. The orange areas corresponded to uncertainty phases where the subject was in contact with the load but without completely carrying it were identified with the video. Inside these phases the external forces prediction with the proposed method was not reliable. Therefore, it was necessary to detect the grip/deposit instants inside these phases. On the presented example, the grip and deposit events predicted by the neural network were consistent with the reference. In particular, all of them were inside the uncertainty phases.

By considering all the motions (156 elementary movements), 8 predicted grip or deposit events were outside the uncertainty areas (the average duration of the grip uncertainty period is $0.33 \mathrm{~s}(0.15)$ and $0.46 \mathrm{~s}(0.18)$ for the deposit uncertainty period). It corresponds to $2.5 \%$ of the 312 events to predict. For these 8 events, the interval between the predicted event and the uncertainty area was averaged to $0.04 \mathrm{~s}( \pm 0.03)$. This error is low compared to the duration of the studied movements (between 4.8 and 10 seconds), which means that these events were close to the uncertainty area.

The chosen descriptors of the grip and deposit moments are therefore satisfactory and the method seems relevant and robust to get the current state of the task (carrying the load or not).

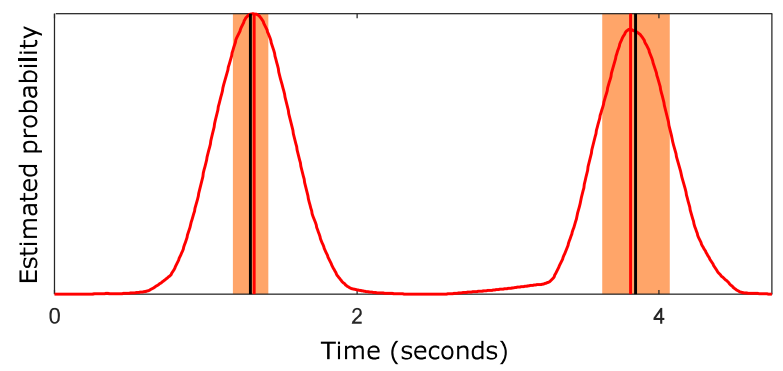

Figure 5: Representative example of the identification of grip and deposit events results as a function of time for one elementary movement. Red curve represents the estimated probability to have a grip or a deposit event. Events identified by a vertical red line are the events of a grip and a deposit estimated with this curve. Events identified by a vertical black line are those defined by using the video, considered as the reference. The orange areas represent the uncertainty phases (also identified through the video).

\section{B. Load motion reconstruction}

The RMSE of the reconstructed coordinate system of the load was $4.7 \mathrm{~cm}( \pm 1.3)$ for its location and $4.2^{\circ}( \pm 2.0)$ for its orientation, in comparison to the marker-based coordinate system. The distance error seemed important compared to the load dimensions. This is probably because, for the asymmetric tasks studied here, the grip differed between the two hands. The geometrical center of the load was not necessarily in the center of the two hand markers. Assumptions made about the orientation of the load seem satisfactory since the mean error was lower than $5^{\circ}$. For the purpose of this study, the load motion reconstruction is an intermediate result for the prediction of contact forces. Thus, the influence of these errors are discussed in the following part of the paper.

\section{External efforts prediction}

A representative example of predicted and measured GRF\&M and LCF is presented Figure 6. It corresponds to one elementary movement of one subject. The relative error was the lowest on the vertical GRF which corresponds to the weight component. The weight transfer was well detected and estimated during the asymmetric task where all GRF were successively applied on each foot. The results in the carrying phase (between the two orange areas) seemed not to be affected by the contact efforts on the hands. Thus, vertical LCF was well predicted on feet. 

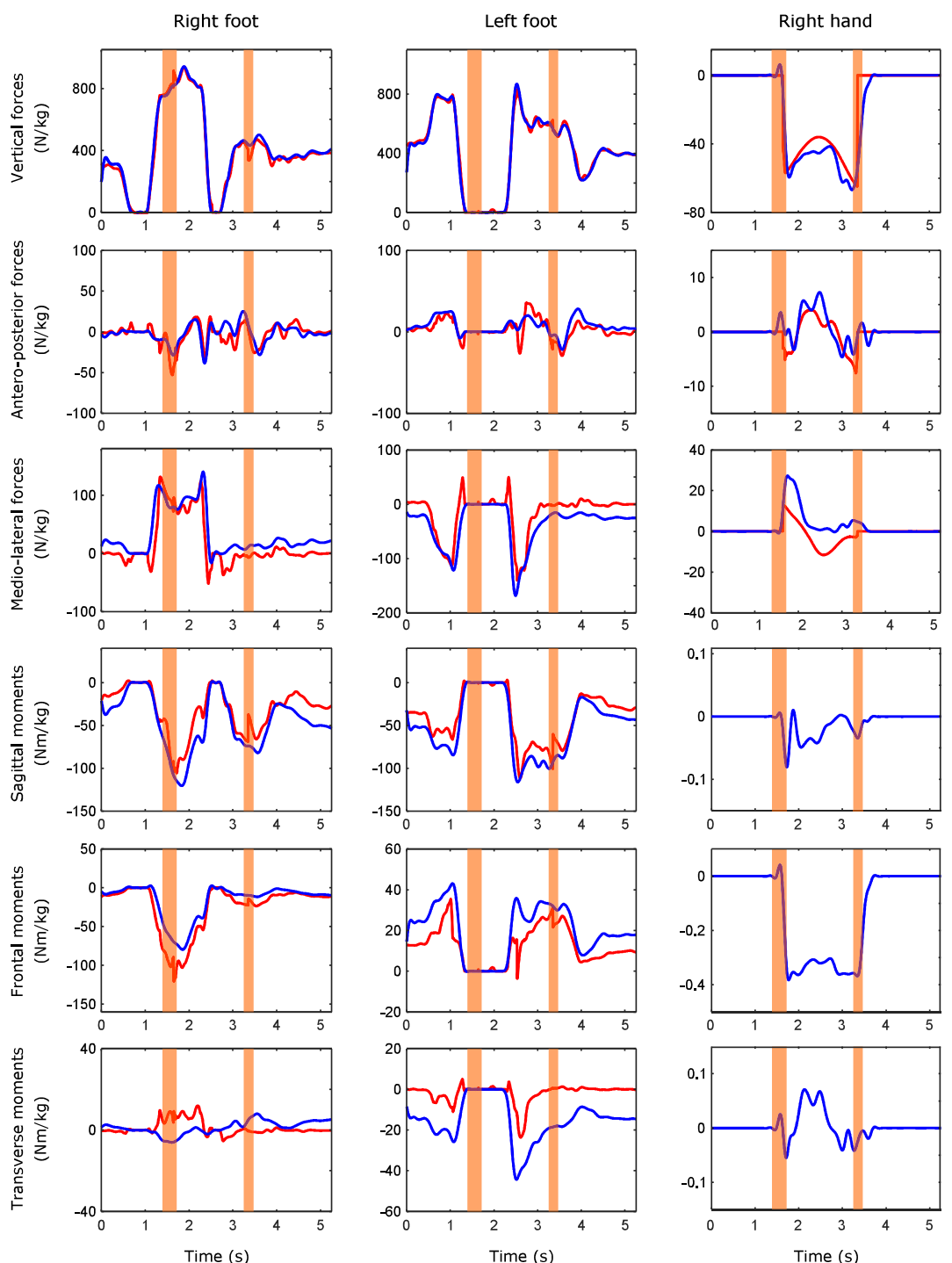

Figure 6: Representative example of predicted and measured GRF\&M and LCF (this elementary movement consisted in carrying the load from area 3 to area 1 with an additional load of $3 \mathrm{~kg}$ ). The blue curve corresponds to the measured data, the red one corresponds to the predicted data. The orange areas represent the uncertainty phases (identified with the video). Inside these areas, the subject was in contact with the load but without completely carrying it.

The most important errors were observed on the mediolateral forces. Concerning the forces on the feet, an offset was identified between the predicted and the measured data on more static phases: the predicted forces were close to zero whereas the measured forces were positive for the right foot and negative for the left. This is probably due to the fact that the subject generated some forces in this direction to improve his stability. As these efforts had no influence on his motion, the proposed method did not predict them. This trend is also visible on hands where the subject applied traction or compression forces to better grip the load. In the same manner, these forces were impossible to predict without additional sensors. This phenomenon had also been observed in the literature by [10] for deep squatting where correlation coefficient for the antero-posterior and medio-lateral forces were between 0.2 and 0.3 .

Concerning the uncertainty phases, higher errors appeared (particularly visible on the vertical GRF under 
the right foot (Figure 6)). In this phase, the subject had indirectly a contact with the ground or the table where the load was placed. This contact was not taken into account in the proposed method, generating prediction errors. Moreover, the low values of the measured sagittal LCM indicates that there was very few load rotation around the axis defined by the two hands. This seems to confirm the assumption made for the load motion reconstruction.

The Table I shows the mean RMSE and rRMSE between measured and predicted GRF\&M and LCF for all the handling tasks of all the subjects. Considering the phases where no load were carried, the results can be compared to the literature since only GRF\&M were considered. The RMSE for the vertical forces was $17.9 \mathrm{~N}$ and $20.3 \mathrm{~N}$ for each foot, corresponding to $0.24 \mathrm{~N} / \mathrm{kg}$ and $0.28 \mathrm{~N} / \mathrm{kg}$. These errors were lower than those presented in the literature for walking motions, whatever the prediction method: empirical functions [6] $(0.90 \mathrm{~N} / \mathrm{kg})$, machine learning [7] $(0.73 \mathrm{~N} / \mathrm{kg})$ or a contact model [10] (between $0.52 \mathrm{~N} / \mathrm{kg}$ and $0.91 \mathrm{~N} / \mathrm{kg}$ ). The RMSE for the anteroposterior axis was below $0.10 \mathrm{~N} / \mathrm{kg}$ since the efforts values were very low due to the performed tasks. The medio-lateral forces component obtained the most important errors $(0.37 \mathrm{~N} / \mathrm{kg}$ and $0.45 \mathrm{~N} / \mathrm{kg}$ for each foot corresponding to $34.8 \%$ and $40.7 \%$ ) which confirms the observations previously made (Figure 6). Also, the GRM were in the same order of magnitude as those reported previously [10]. Considering all these results for the phases with no load, one can conclude that the method presented here reproduces similar results as in the literature in similar cases.

Considering the phases with load, the RMSE for the GRF\&M were quite similar. The prediction method previously validated only for the GRF\&M was adapted to the addition of hand contact and for asymmetric handling tasks. Consideration of the hands contact did not introduce additional error on GRF\&M prediction. The RMSE for the vertical axis of the LCF was $0.86 \mathrm{~N} / \mathrm{kg}$ and $0.50 \mathrm{~N} / \mathrm{kg}$ according to the load carried. These results are in the same order of magnitude as the errors found for the GRF. However, as the load mass is relatively light, the absolute error was below $6 \mathrm{~N}$. In the same manner as for the GRF, the most important errors were reported on the medio-lateral forces $(12.6 \mathrm{~N}$ and $16.9 \mathrm{~N}$ according to the load carried). These forces correspond to traction or compression forces applied by the subject to better grip the load. The higher error observed with the additional load could be due to the fact that the subject applied a more important force along the medio-lateral axis to ensure the stability of the load during the motion. Even if the load motion reconstruction error is not negligible, prediction results are satisfactory.

\section{L5/S1 joint moments estimation}

The RMSE error on the L5/S1 joint moments estimation were $18.7 \mathrm{Nm}( \pm 11), 10.6 \mathrm{Nm}( \pm 7.8)$ and $12.6 \mathrm{Nm}$ $( \pm 9.6)$ on sagittal, frontal and transverse axis, respectively. The associated rRMSE are $9.9 \%( \pm 5.8), 8.9 \%$ $( \pm 6.5)$ and $37 \%( \pm 36)$. By comparing a bottom-up and a top-down approach during handling tasks, Larivière et al. [25] reported uncertainties of $15 \mathrm{Nm}, 8 \mathrm{Nm}$ and $8 \mathrm{Nm}$ for the L5/S1 joint moment estimation on sagittal, frontal and transverse axes, respectively. For a similar study, Plamondon et al. [26] reported uncertainties of $4 \mathrm{Nm}, 4 \mathrm{Nm}$ and $5 \mathrm{Nm}$ along the same axes. Finally, Kingma et al. [27] observed uncertainties ranging from 3.9 $\mathrm{Nm}$ to $23.7 \mathrm{Nm}$ on sagittal axis, $5.4 \mathrm{Nm}$ to $20.5 \mathrm{Nm}$ on frontal axis and $1.9 \mathrm{Nm}$ to $15.2 \mathrm{Nm}$ on transverse axis. The observed errors in our study are higher than uncertainties reported in the literature. However, they are of the same order of magnitude. Moreover, for the sagittal axis, the relative error was lower than $10 \%$. This axis contains the most important moment values and is the most studied in handling tasks assessment in the literature. More important relative error was observed on the transverse axis where the moment values were lower.

Finally, the current method estimated the GRF\&M and the LCF during asymmetric handling tasks with a small margin of error, and with the subject motion as a unique source of experimental data. This estimation could be used to compute kinetics variables as back loading with an acceptable error. Thanks to this method, inverse dynamics studies are not limited anymore to motions where the body is in contact with force sensors to estimate the external forces. It may be extremely useful in work tasks assessment or sport gesture analyses, opening a wide range of applications. It indicates that we can develop and analyze more complex experimental protocols with a higher level of generalizability to real tasks in a motion analysis lab.

\section{E. Limits and perspectives}

In the current study, the prediction was based on several parameters as the number of contact points, the thresholds distance and velocity, the maximal force available at each point, the friction coefficient between the feet and the ground and the standard deviation used in the 
Table I: Mean RMSE and rRMSE between measured and predicted GRF\&M and LCF for all the handling tasks of all subjects. Results for each component were divided into 3 parts: the subject did not carry the load, the subject carried the load without additional load and the subject carried the load with an additional load of $3 \mathrm{~kg}$. LCF for the first phase are not presented since they were zero and LCM are not presented (both denoted by *).

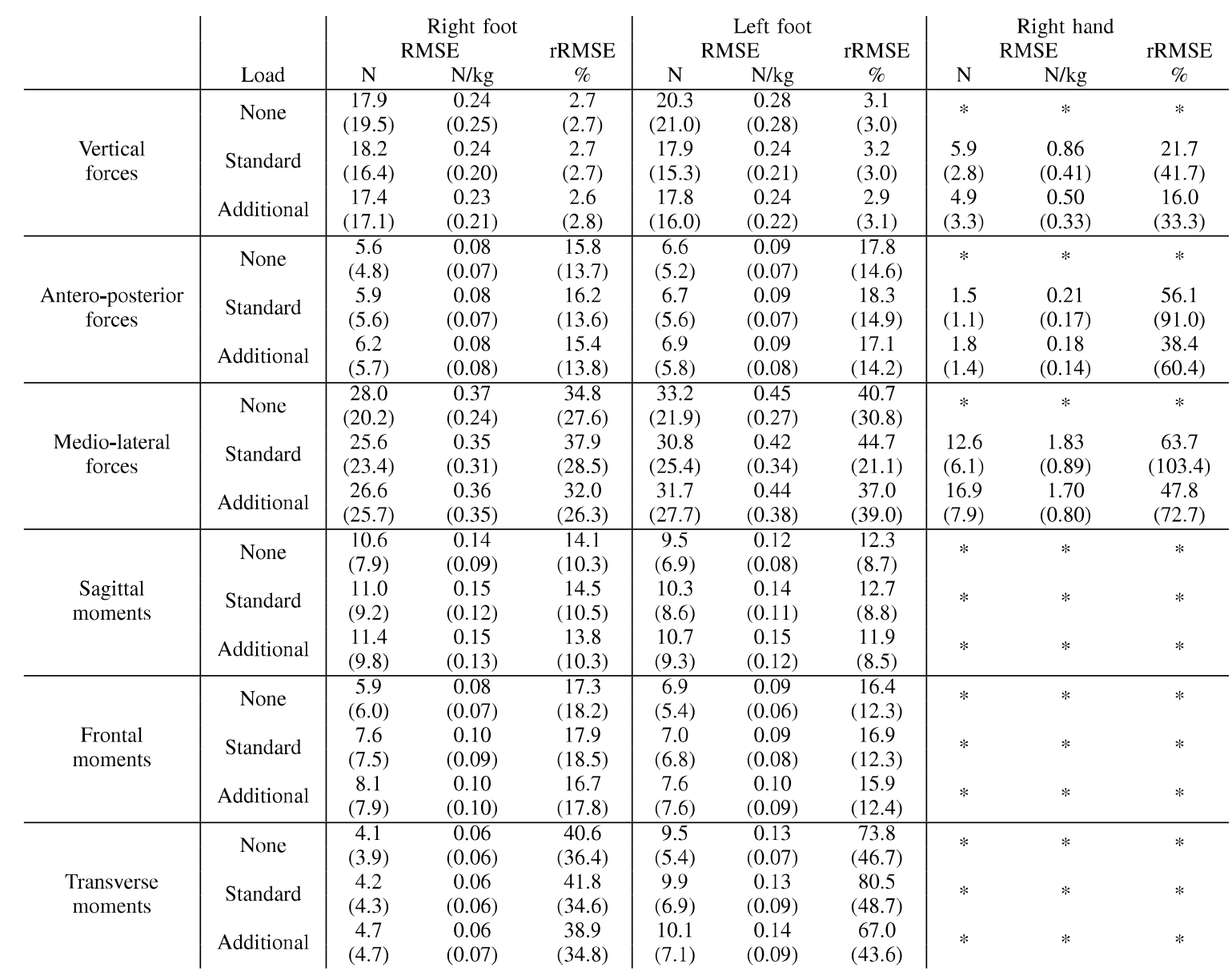

neural network. These parameters were inspired by the literature and adapted for this work. Even if this set of parameters give satisfactory results, a parametric study may be useful to improve the prediction results and increase the robustness of the method. In this spirit, Fluit et al. [10] conducted a sensitivity analysis on the thresholds distance and velocity and the maximal force available at each point. It consisted in varying the different values at $70 \%$ and $130 \%$ of their original magnitude and observing the prediction results. Such an approach may be applied to the current study. In the same manner, the locations of the contact points have an impact on the method. In this study, for each foot and for each hand, all of the contact points of a given solid were included in a unique plane (corresponding to a flat foot and an extended hand). An evolution of these contact points definitions - adding degrees of freedom or following more accurately the osteoarticular structure, could probably improve the contact model.

The proposed method was based on the subject motion obtained by an optoelectronical motion capture system. However, this kind of system is mostly available in motion analysis laboratory. In the ideal case, ergonomics studies are performed directly in the workplace. To this end, wearable motion capture systems may be used. Inertial motion capture system (as the most commonly used XSens [28]) permits already to evaluate the handler kinematics [29]-[31]. Depth cameras are also proposed for workstation ergonomic postures assessments [32], [33]. In the laboratory, GRF\&M are predicted by using these wearable systems by using empirical functions 
[34]-[36] or by using a contact models on walking motions [37]-[39]. The method proposed in this paper could be extended to wearable motion capture systems and thus be used to evaluate kinetics data in the workplace.

The identification of the grip and deposit events has already been mentioned in the literature as a limitation to predict the external forces. In most cases, a force platform allows these events to be identified [14], [35]. The use of a neural network proposed in this work permitted their identification with the subject motion only. However, elementary movements studied in this experimentation were imposed for the subject and were standardized, explaining also that the neural network had such great performances. In a workplace, handlers have more freedom to perform their motions. It implies that the grip and deposit moments are more difficult to predict with variable loads without handle. The addition of sensors as instrumented shoes [35], [40], [41] could allow to improve the external efforts estimation by detecting directly contact phases instead of learning them. Combining learning and measures may also be an interesting perspective to solve this issue.

Moreover, pre-grip phases are common in handling tasks. In these phases (corresponding to the uncertainty phases in this paper), the motion-based prediction method is not efficient. Added sensors seems necessary if pre-grips are specifically studied.

Finally, BSIP were here based on anthropometric tables. These parameters influence directly the external efforts estimation, thus a subject-specific estimation of the BSIP should improve their prediction [42] (particularly with subjects far from standard anthropometrics). Calibration techniques based on classical devices available in any motion analysis laboratory were developed to improve the BSIP estimation by using forces platforms [43], [44] or image-based techniques [45]. Such methods could be associated to the external efforts prediction method to enhance its efficiency.

\section{CONCLUSION}

The results of the current study showed that a prediction method based on a contact model allowed external efforts to be predicted on asymmetric handling tasks with only the subject motion. GRF\&M, LCF and L5/S1 joint moments were estimated with a small margin of error. The prediction method enabled to quantitatively assess handling tasks on the basis of kinetics variables without additional instrumentation as force sensors. The workers follow-up could thus be improved by assessing the ergonomics of simulated tasks more representative of those performed in the workplace.

\section{REFERENCES}

\section{REFERENCES}

[1] A. Parent-Thirion, I. Biletta, J. Cabrita, O. Vargas Llave, G. Vermeylen, A. Wilczyńska, and M. Wilkens, "Combining data from different surveys in analysis: Compatibility of the 2013 european company survey and the 2015 european working conditions survey,” Tech. Rep., 2017.

[2] M. Gagnon, "The efficacy of training for three manual handling strategies based on the observation of expert and novice workers," Clinical Biomechanics, vol. 18, no. 7, pp. 601-611, 2003.

[3] W. S. Marras, S. A. Lavender, S. A. Ferguson, R. E. Splittstoesser, G. Yang, and P. Schabo, "Instrumentation for measuring dynamic spinal load moment exposures in the workplace," Journal of Electromyography and Kinesiology, vol. 20, no. 1, pp. 1-9, 2010.

[4] A. Plamondon, D. Denis, A. Delisle, C. Larivière, E. Salazar, I. M. research group et al., "Biomechanical differences between expert and novice workers in a manual material handling task," Ergonomics, vol. 53, no. 10, pp. 1239-1253, 2010.

[5] B. Koopman, H. J. Grootenboer, and H. J. De Jongh, "An inverse dynamics model for the analysis, reconstruction and prediction of bipedal walking," Journal of biomechanics, vol. 28, no. 11, pp. 1369-1376, 1995

[6] E. J. Dijkstra and E. M. Gutierrez-Farewik, "Computation of ground reaction force using zero moment point," Journal of biomechanics, vol. 48, no. 14, pp. 3776-3781, 2015.

[7] A. Choi, J.-M. Lee, and J. H. Mun, "Ground reaction forces predicted by using artificial neural network during asymmetric movements," International Journal of Precision Engineering and Manufacturing, vol. 14, no. 3, pp. 475-483, 2013.

[8] S. E. Oh, A. Choi, and J. H. Mun, "Prediction of ground reaction forces during gait based on kinematics and a neural network model," Journal of biomechanics, vol. 46, no. 14, pp. 2372-2380, 2013.

[9] W. R. Johnson, A. Mian, C. J. Donnelly, D. Lloyd, and J. Alderson, "Predicting athlete ground reaction forces and moments from motion capture," Medical \& biological engineering \& computing, pp. 1-12, 2018.

[10] R. Fluit, M. S. Andersen, S. Kolk, N. Verdonschot, and H. F. Koopman, "Prediction of ground reaction forces and moments during various activities of daily living," Journal of biomechanics, vol. 47, no. 10, pp. 2321-2329, 2014.

[11] Y. Jung, M. Jung, J. Ryu, S. Yoon, S.-K. Park, and S. Koo, "Dynamically adjustable foot-ground contact model to estimate ground reaction force during walking and running," Gait \& posture, vol. 45 , pp. 62-68, 2016

[12] S. Skals, M. K. Jung, M. Damsgaard, and M. S. Andersen, "Prediction of ground reaction forces and moments during sportsrelated movements," Multibody system dynamics, vol. 39, no. 3, pp. 175-195, 2017.

[13] T. Robert, J. Causse, and G. Monnier, "Estimation of external contact loads using an inverse dynamics and optimization approach: general method and application to sit-to-stand maneuvers," Journal of biomechanics, vol. 46, no. 13, pp. 2220-2227, 2013.

[14] V. Bonnet, C. Azevedo-Coste, T. Robert, P. Fraisse, and G. Venture, "Optimal external wrench distribution during a multi-contact sit-to-stand task," IEEE Transactions on Neural Systems and Rehabilitation Engineering, vol. 25, no. 7, pp. 987-997, 2017. 
[15] A. S. Koopman, I. Kingma, G. S. Faber, J. Bornmann, and J. H van Dieën, "Estimating the $15 \mathrm{~s} 1$ flexion/extension moment in symmetrical lifting using a simplified ambulatory measuremen system," Journal of biomechanics, vol. 70, pp. 242-248, 2018.

[16] G. Wu, S. Siegler, P. Allard, C. Kirtley, A. Leardini, D. Rosenbaum, M. Whittle, D. D D'Lima, L. Cristofolini, H. Witte et al., "Isb recommendation on definitions of joint coordinate system of various joints for the reporting of human joint motion-part $\mathrm{i}$ ankle, hip, and spine," Journal of biomechanics, vol. 35 , no. 4 , pp. 543-548, 2002.

[17] G. Wu, F. C. Van der Helm, H. D. Veeger, M. Makhsous, P. Van Roy, C. Anglin, J. Nagels, A. R. Karduna, K. McQuade, $\mathrm{X}$. Wang et al., "Isb recommendation on definitions of joint coordinate systems of various joints for the reporting of human joint motion-part ii: shoulder, elbow, wrist and hand," Journal of biomechanics, vol. 38, no. 5, pp. 981-992, 2005.

[18] D. A. Winter, Biomechanics and motor control of human move ment. John Wiley \& Sons, 2009.

[19] A. Muller, C. Germain, C. Pontonnier, and G. Dumont, "A simple method to calibrate kinematical invariants: application to overhead throwing," in ISBS-Conference Proceedings Archive, vol. 33, no. 1, 2016

[20] R. Dumas, L. Cheze, and J.-P. Verriest, "Adjustments to mcconville et al. and young et al. body segment inertial parameters,' Journal of biomechanics, vol. 40, no. 3, pp. 543-553, 2007.

[21] T.-W. Lu and J. O'connor, "Bone position estimation from skin marker co-ordinates using global optimisation with joint constraints," Journal of biomechanics, vol. 32, no. 2, pp. 129 134, 1999.

[22] R. Featherstone, Rigid body dynamics algorithms. Springer 2014.

[23] L. Ren, R. K. Jones, and D. Howard, "Whole body inverse dynamics over a complete gait cycle based only on measured kinematics," Journal of biomechanics, vol. 41, no. 12, pp. 2750 2759, 2008.

[24] A. Muller, C. Pontonnier, P. Puchaud, and G. Dumont, "CusToM a Matlab toolbox for musculoskeletal simulation," Journal of Open Source Software, vol. 4, no. 33, pp. 1-3, 2019.

[25] C. Larivière and D. Gagnon, "Comparison between two dynamic methods to estimate triaxial net reaction moments at the $15 / \mathrm{s}$ joint during lifting," Clinical Biomechanics, vol. 13, no. 1, pp. 36-47, 1998.

[26] A. Plamondon, M. Gagnon, and P. Desjardins, "Validation of two 3-d segment models to calculate the net reaction forces and moments at the $15 \mathrm{~s} 1$ joint in lifting," Clinical Biomechanics, vol. 11, no. 2, pp. 101-110, 1996.

[27] I Kingma, M. P de Looze, H. M. Toussaint, H G. Klijnsma, and T. B. Bruijnen, "Validation of a full body 3-d dynamic linked segment model," Human Movement Science, vol. 15, no. 6, pp. 833-860, 1996.

[28] D. Roetenberg, H. Luinge, and P. Slycke, "Xsens mvn: full 6dof human motion tracking using miniature inertial sensors," Xsens Motion Technologies BV, Tech. Rep, vol. 1, 2009.

[29] G. S. Faber, I. Kingma, S. M. Bruijn, and J. H. van Dieën, "Optimal inertial sensor location for ambulatory measurement of trunk inclination," Journal of biomechanics, vol. 42, no. 14, pp. 2406-2409, 2009

[30] N. Vignais, M. Miezal, G. Bleser, K. Mura, D. Gorecky, and F. Marin, "Innovative system for real-time ergonomic feedback in industrial manufacturing," Applied ergonomics, vol. 44, no. 4, pp. 566-574, 2013.

[31] X. Robert-Lachaine, H. Mecheri, C. Larue, and A. Plamondon, "Validation of inertial measurement units with an optoelectronic system for whole-body motion analysis," Medical \& biological engineering \& computing, vol. 55, no. 4, pp. 609-619, 2017.

[32] P. Plantard, A. Muller, C. Pontonnier, G. Dumont, H. P. Shum, and F. Multon, "Inverse dynamics based on occlusion-resistant kinect data: Is it usable for ergonomics?" International Journal of Industrial Ergonomics, vol. 61, pp. 71-80, 2017.
[33] P. Plantard, H. P. Shum, A.-S. Le Pierres, and F. Multon, "Validation of an ergonomic assessment method using kinect data in real workplace conditions," Applied ergonomics, vol. 65, pp. $562-569,2017$

[34] A. Karatsidis, G. Bellusci, H. M. Schepers, M. de Zee, M. S. Andersen, and P. H. Veltink, "Estimation of ground reaction forces and moments during gait using only inertial motion capture," Sensors, vol. 17, no. 1, p. 75, 2016

[35] G. Faber, C. Chang, I. Kingma, J. Dennerlein, and J. van Dieën, "Estimating 3d 15/s1 moments and ground reaction forces during trunk bending using a full-body ambulatory inertial motion capture system,' Journal of biomechanics, vol. 49, no. 6, pp. 904 912, 2016.

[36] R. D. Gurchiek, R. S. McGinnis, A. R. Needle, J. M. McBride, and H. van Werkhoven, "The use of a single inertial sensor to estimate 3-dimensional ground reaction force during accelerative running tasks," Journal of biomechanics, vol. 61, pp. 263-268, 2017

[37] M. Aurbach, K. Wagner, F. Süß, and S. Dendorfer, "Implementation and validation of human kinematics measured using imus for musculoskeletal simulations by the evaluation of joint reaction forces," in CMBEBIH 2017. Springer, 2017, pp. 205-211.

[38] M. Eltoukhy, C. Kuenze, M. S. Andersen, J. Oh, and J. Signorile, "Prediction of ground reaction forces for parkinson's disease patients using a kinect-driven musculoskeletal gait analysis model," Medical Engineering and Physics, vol. 50, pp. 75-82, 2017.

[39] A. Karatsidis, M. Jung, H. M. Schepers, G. Bellusci, M. de Zee, P. H. Veltink, and M. S. Andersen, "Musculoskeletal modelbased inverse dynamic analysis under ambulatory conditions using inertial motion capture," Medical Engineering \& Physics, 2019.

[40] Y. Jung, M. Jung, K. Lee, and S. Koo, "Ground reaction force estimation using an insole-type pressure mat and joint kinematics during walking," Journal of biomechanics, vol. 47, no. 11, pp. 2693-2699, 2014

[41] P. G. Rosquist, G. Collins, A. J. Merrell, N. J. Tuttle, J. B. Tracy, E. T. Bird, M. K. Seeley, D. T. Fullwood, W. F. Christensen, and A. E. Bowden, "Estimation of 3d ground reaction force using nanocomposite piezo-responsive foam sensors during walking," Annals of biomedical engineering, vol. 45, no. 9, pp. 2122-2134, 2017.

[42] C. Hansen, G. Venture, N. Rezzoug, P. Gorce, and B. Isableu, "An individual and dynamic body segment inertial parameter validation method using ground reaction forces," Journal of biomechanics, vol. 47, no. 7, pp. 1577-1581, 2014.

[43] G. Venture, K. Ayusawa, and Y. Nakamura, "Identification of human mass properties from motion," IFAC Proceedings Volumes, vol. 42, no. 10, pp. 988-993, 2009.

[44] A. Muller, D. Haering, C. Pontonnier, and G. Dumont, "Noninvasive techniques for musculoskeletal model calibration," in Congrès Français de Mécanique, 2017

[45] H. Pillet, X. Bonnet, F. Lavaste, and W. Skalli, "Evaluation of force plate-less estimation of the trajectory of the centre of pressure during gait. comparison of two anthropometric models," Gait \& posture, vol. 31, no. 2, pp. 147-152, 2010. 